\title{
POTTS MODELS and RELATED PROBLEMS in STATISTICAL MECHANICS
}




\section{SERIES ON ADVANCES IN STATISTICAL MECHANICS}

Editor-in-Chief: M. Rasetti

Published

Vol. 1: Integrable Systems in Statistical Mechanics edited by G. M. D'Ariano, A. Montorsi \& M. Rasetti

Vol. 2: Modern Methods in Equilibrium Statistical Mechanics by M. Rasetti

Vol. 3: Progress in Statistical Mechanics edited by C. K. Hu

Vol. 4: Thermodynamics of Complex Systems by L. Sertorio 


\section{POTTS MODELS and RELATED PROBLEMS in STATISTICAL MECHANICS}

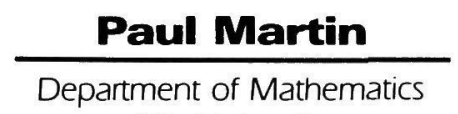

City University 
Published by

World Scientific Publishing Co. Pte. Ltd.

P O Box 128, Farrer Road, Singapore 9128

USA office: 687 Hartwell Street, Teaneck, NJ 07666

UK office: 73 Lynton Mead, Totteridge, London N20 8DH

Library of Congress Cataloging-in-Publication data is available.

\section{POTTS MODELS AND RELATED PROBLEMS \\ IN STATISTICAL MECHANICS}

Copyright @ 1991 by World Scientific Publishing Co. Pte. Ltd.

All rights reserved. This book, or parts thereof, may not be reproduced in any form or by any means, electronic or mechanical, including photocopying, recording or any information storage and retrieval system now known or to be invented, without written permission from the Publisher.

ISBN 981-02-0075-7

Printed in Singapore by JBW Printers \& Binders Pte. Ltd. 


\section{Preface}

This book is largely a rearrangement of a set of lectures I gave at RIMS, Kyoto in spring 1989. The additional material is either from lectures I attended in that period, or arose in the process of rearrangement. The book itself was written at Birmingham University Mathematics Department (summer 89) and City University Mathematics Department (autumn 89 onward).

The central theme of Potts models was chosen with the aim of drawing together, in a systematic way, some key ideas from the continuing explosion of statistical mechanical research. The acid test of relevance to this well established physical picture is one sure way to force a coherent direction on the general explosion of results.

The potential drawback of writing a book on such a vital subject is that one feels more and more pressure each week to incorporate the very latest results. Succumbing to this pressure would obviously result in a rather slowly converging process. In any case, most up to the minute research turns out not, on mature reflection, to be suitable for a textbook. I therefore introduced a cut-off on new material after spring 1989 (except where directly pertinent to the presentation of material already included). At time of going into print no aspect of the subject which has matured since then seems, by its omission, to leave a gap in the presentation.

Of course the subject will soon move on, but the fundamentals will still be fundamental. With this in mind, another aim was to provide a handy work of reference for my own research use, just as Baxter's (1982) book (with a different slant and broader but less recent perspective) has become for so many.

I have tried to make the mathematical development as explicit as is consistent with a finite number of pages. As a physicist I have in particular strived to convey some of the pleasure I have found in learning and applying algebraic techniques to physical problems... and physics is forever trying to teach us algebra.

London, England

Paul Martin 
This page is intentionally left blank 


\section{Acknowledgments}

Large debts of gratitude are due to B W Westbury, T Miwa, M Jimbo, A Kuniba, M Okado, J B Martin, A J McKane, G P McCauley, M B Green, R A Wilson, G P Launer, M M Martin, C J B Martin and P E Woodley. Thanks are also due to $M$ Wadati, $T$ Deguchi, A J Guttman, E Date, Y Yamada, H N V Temperley and R Curtis. 
This page is intentionally left blank 


\section{Contents}

Preface

Acknowledgements

vii

1 Introduction 1

1.1 On layout and objectives . . . . . . . . . . . . 2

1.2 Statistical mechanics ................. 6

1.2.1 Partition functions and Hamiltonians . . . . . . 7

1.2 .2 Defining lattices . . . . . . . . . . . . . 10

1.2.3 Other statistical mechanical functions . . . . . . 15

1.3 Potts models . . . . . . . . . . . . . . . . . . . 16

1.4 Phase transitions . . . . . . . . . . . . . . . . 17

1.4.1 Order parameters . . . . . . . . . . . . . 18

1.4.2 Critical exponents . . . . . . . . . . . . 20

1.5 Dichromatic polynomials . . . . . . . . . . . . . 21

1.6 High and low temperature series ............ 23

1.7 Block spin renormalisation . . . . . . . . . . . . . . . 24

1.7.1 Fixed points . . . . . . . . . . . . 31

2 Transfer matrices $\quad 35$

2.1 Partition vectors . . . . . . . . . . . . . . 36

2.1.1 On internalising a common boundary . . . . . 39

2.1.2 Local transfer matrices . . . . . . . . . . . . . . . 41

2.2 Algebraic formulation . . . . . . . . . . . . . . 45

2.2.1 General local interactions . . . . . . . . . 46

2.2 .2 Transfer matrix algebras . . . . . . . . . . . . . . 47

2.3 Automorphisms of the lattice . . . . . . . . . . . . . . . 48

2.4 Perron Frobenius theorem . . . . . . . . . . . . . . . . . 49

2.5 The free energy . . . . . . . . . . . . . . . 50

2.6 Correlation functions . . . . . . . . . . . . . . 52

2.7 Spectrum of the transfer matrix . . . . . . . . . . 54

2.8 Potts models . . . . . . . . . . . . . . . . . 55

2.9 Standard transfer matrices and duality . . . . . . . . . 56

2.9.1 Alternative layering directions . . . . . . . . 59 
2.10 Spectrum inversion transformations . . . . . . . 63

3 On commuting transfer matrices $\quad 67$

3.1 Yang-Baxter equations . . . . . . . . . . . . 68

3.1.1 Commuting layer transfer matrices . . . . . . . 69

3.2 Algebraic consequences of the relations . . . . . . . . . 72

3.2.1 The braid point . . . . . . . . . . . . 72

3.2 .2 Translations revisited . . . . . . . . 75

3.3 Alternative layering directions . . . . . . . . . . 76

3.4 The two dimensional Ising model . . . . . . . . . 77

3.4.1 Useful transfer matrix identities for $Q=2 \ldots \ldots$

3.4.2 Applying the inversion relation . . . . . . . . 82

3.4.3 Reparameterised YB equation . . . . . . . . . 83

4 On exactly solved cases $\quad 89$

4.1 The two dimensional Ising model . . . . . . . . . . . 89

4.1.1 Solution by rotations . . . . . . . . . . . 90

4.1.2 By translation in the layer . . . . . . . . . . 95

4.1.3 By translation in the plane .......... 100

4.2 On conformal field theory . . . . . . . . . . . . . 101

5 Algebra: general principles $\quad 107$

5.1 Algebras . . . . . . . . . . . . . . . 107

5.1 .1 On specifying algebras . . . . . . . . . . 108

5.1.2 Subalgebras and quotients . . . . . . . . . 110

5.1.3 Linear representation theory .......... 112

5.2 Physics and the radical . . . . . . . . . . . . . . . 114

5.3 Induction and restriction . . . . . . . . . . . . . . 115

5.4 On the structure of algebras . . . . . . . . . . . . 120

5.4 Morita equivalence . . . . . . . . . . . 121

5.5 Centraliser algebras ................. 123

5.6 Bialgebras . . . . . . . . . . . . . . . . 123

5.7 Algebraic overview of following chapters . . . . . . . . 125

5.7 .1 On the braid group . . . . . . . . . . 127

5.7 .2 Algebra morphisms . . . . . . . . . . 128

6 Temperley-Lieb algebras: generic cases 131

6.1 Review . . . . . . . . . . . . . . . . . 132

6.1 .1 Technical notes .................. 133

6.2 Preliminary remarks . . . . . . . . . . . . . . 134

6.2.1 Two faithful diagrammatic representations . . . 136

6.3 Generic structure of $T_{k}(q) \ldots \ldots \ldots$. . . . . . 140 
6.3.1 Combinatorial identities . . . . . . . . . . . 141

6.3.2 Sequence notation . . . . . . . . . . . . . . 142

6.3.3 A primitive central idempotent in $T_{n}(q) \ldots \ldots . .147$

6.3.4 Translation/reflection notation . . . . . . . . . 149

6.4 Bases for $T_{n}(q) \ldots \ldots \ldots \ldots \ldots \ldots$

6.5 Useful identities . . . . . . . . . . . . . . . 158

6.5 .1 The word $X(d, c) \ldots \ldots \ldots \ldots 158$

6.5.2 Longest words, module by module . . . . . . . . . 161

6.5.3 The ABF regular representation ... . . . . . . 164

$\begin{array}{llr}7 & \text { Special cases } & 167\end{array}$

7.1 More combinatorics and sequences ........... 167

7.2 Towards the main theorem . . . . . . . . . . . . 169

7.2 .1 Indexing $T_{n}(q)$ modules $\ldots \ldots \ldots 171$

7.3 The main theorem . . . . . . . . . . . . . . 171

7.4 Proof of main theorem . . . . . . . . . . . . . 174

8 Graph Temperley-Lieb algebras 209

8.1 Introduction . . . . . . . . . . . . . . . . . . 209

8.2 The Potts Representation . . . . . . . . . . . . . . 211

8.2 .1 Construction . . . . . . . . . . . . . 211

8.2.2 The Potts quotient algebra . . . . . . . . . . 212

8.2.3 Reducibility of the Potts representation. . . . . . . . 213

8.3 Partition representations . . . . . . . . . . . . . . . 214

8.3.1 The bases . . . . . . . . . . . . . . . . . 214

8.3.2 Computation of $\mathcal{S}_{n}(i) \ldots \ldots \ldots \ldots \ldots$

8.3.3 Representation type $1 \ldots \ldots \ldots 216$

8.3.4 Representation type $2 \ldots \ldots . \ldots 217$

8.3.5 Fixed $n$ pregraph dependence of type 2 bases. . . . . 222

8.3.6 Generic irreducibility of type 2 representations. . . . 223

8.3.7 Quotient relations for type 2 representations. . . . . 225

8.4 Physical consequences. . . . . . . . . . . . . . . 226

8.5 Quotient relations: the Potts representation . . . . . . . . 227

9 Hecke Algebras $\quad 231$

9.1 Review . . . . . . . . . . . . . . . . . . 231

9.1.1 Technical notes . . . . . . . . . . . . 232

9.2 On the structure of $H_{k}(q) \ldots \ldots \ldots \ldots \ldots \ldots$

9.2.1 A primitive central idempotent in $H_{n}(q) \ldots \ldots 234$

9.2.2 Another primitive central idempotent . . . . . . 236

9.2.3 The quotient algebras $N H_{n}(q) \ldots \ldots . \ldots 237$

9.2.4 The word problem ............. 237 
9.2 .5 Sequence notation . . . . . . . . . . . . . . 240

9.3 On bases for $H_{n}(q) \ldots \ldots \ldots \ldots \ldots$

9.3.1 A basis for $\alpha$-permutation representations . . . . . 244

9.3.2 On irreducible representations .......... . . 248

9.4 The generic structure of $H_{n}(q) \ldots \ldots \ldots \ldots$

9.5 On the non-generic structure of $H_{n}(q) \ldots \ldots \ldots 256$

9.5.1 On the structure of $N_{n}(q) \ldots \ldots \ldots \ldots$

9.5 .2 On $T_{k}(q)$ modules . . . . . . . . . . . 258

9.5.3 The structure of $2 H_{k}(q) \ldots \ldots \ldots 266$

9.5.4 The structure of $3 H_{k}(q)$ and higher $N \ldots \ldots 267$

9.6 The defining representation of $\mathrm{NH}_{k}(q) \ldots \ldots \ldots \ldots$

10 Algebraic formalism for $Z_{Q}$ symmetry 271

10.1 Introduction . . . . . . . . . . . . . . . 271

10.1.1 Notation. . . . . . . . . . . . . . 272

$10.2 Z_{Q}$ symmetric models . . . . . . . . . . . . 273

10.2.1 Transfer matrices . . . . . . . . . . . . . 274

10.2.2 Simplicial Clifford algebras . . . . . . . . . . 276

10.2.3 Canonical representations . . . . . . . . . . 277

10.3 Temperley-Lieb subalgebras . . . . . . . . . . . . . 278

10.3.1 Lattice gauge model representations . . . . . . . . 278

10.3.2 Quotient relations for the gauge representation . . . 281

11 The modelling of phase transitions $\quad \mathbf{2 8 7}$

11.1 Zeros of the partition function . . . . . . . . . . . . . 287

11.2 Semi-infinite systems . . . . . . . . . . . . . . . . . . . . . . . . . . . . . . . . . . . .

11.3 Finite lattice results . . . . . . . . . . . . . . . . 294

11.4 Energy, entropy and combinatorics . . . . . . . . 295

12 Vertex models and related algebras 309

12.1 Homogeneous 6 vertex model . . . . . . . . . . . . . 309

12.1.1 Vertex algebras ............... . . 309

12.1 .2 Deriving a model . . . . . . . . . . . . . 311

12.1.3 On solving the model ............ 313

12.2 Asymmetric 6 vertex models . . . . . . . . . . . . 317

12.2.1 Inhomogeneous 6 vertex models . . . . . . . . 319

12.2.2 Equivalence with the Potts model . . . . . . . . 319

12.3 Homogeneous $Z_{N}$ vertex models . . . . . . . . . 321 
13 Braids and cables $\quad 323$

13.1 Introduction . . . . . . . . . . . . . . . . . 323

13.1.1 Cabling quotients of $B_{n} \ldots \ldots \ldots 325$

13.2 Cabling Temperley-Lieb algebras . . . . . . . . . . . . 325

13.2.1 The local relation . . . . . . . . . . . . 331

13.2.2 Other idempotents . . . . . . . . . . . 334

$\begin{array}{lr}\text { Bibliography } & \mathbf{3 3 7}\end{array}$

$\begin{array}{ll}\text { Index } & 341\end{array}$ 